\title{
Classic Kaposi Sarcoma
}

National Cancer Institute

\section{Source}

National Cancer Institute. Classic Kaposi Sarcoma. NCI Thesaurus. Code C9112.

A vascular sarcoma that commonly occurs in the lower extremities. It occurs predominantly in elderly male patients of southern European ancestry. It is characterized by the presence of purple, red-blue, or dark brown macular lesions, plaques and nodules. This disease is usually slow growing, although it can spread to the lungs and the gastrointestinal tract. If necessary, cutaneous lesions can be treated with radiation. 